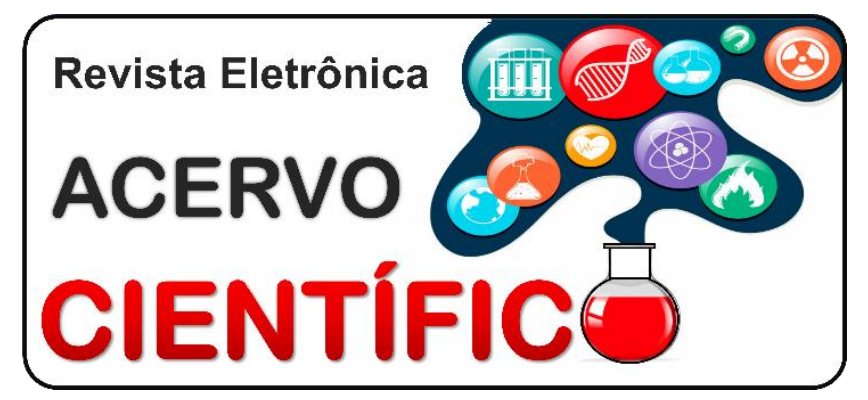

ESTUDO DE CASO

Recebido em: 1/2021

Aceito em: 1/2021

Publicado em: $1 / 2021$

\title{
Pioderma gangrenoso em paciente portadora de doença de Crohn responsivo a terapia biológica com infliximabe: relato de caso no Amazonas
}

\author{
Gangrenous pyoderma in a patient with Crohn's disease responsive to biological therapy \\ with infliximab: case report in Amazonas
}
Pioderma gangrenosa en paciente con enfermedad de Crohn sensible a terapia biológica con infliximab: reporte de caso en Amazonas

\begin{abstract}
Wanderson Assunção Loma ${ }^{1 *}$, Arlene dos Santos Pinto ${ }^{1}$, Ana Beatriz da Cruz Lopo de Figueiredo1, Ketlin Batista de Morais Mendes ${ }^{1}$, Aline de Vasconcellos Costa e Sá Storino ${ }^{1}$, Wilson Marques Ramos Júnior ${ }^{1}$.
\end{abstract}

Resumo: Esse artigo buscou relatar um caso de pioderma gangrenoso responsivo a terapia de resgate com infliximabe. Paciente do sexo feminino, 42 anos, portadora de Doença de Crohn em uso de Mesalazina. Evoluiu com artralgia em grandes articulações, quando foi prescrita Azatioprina e desde então apresentou sinais flogísticos em tecidos moles de ambos os antebraços e diarreia mucossanguinolenta. Necessitou de internação hospitalar, onde realizou inicialmente antibioticoterapia e posteriormente corticoterapia via endovenosa, sem melhora do quadro clínico. No final da segunda semana de tratamento, as lesões cutâneas tornaram-se bem características de pioderma gangrenoso e ainda apresentava diarreia. Foi iniciada terapia biológica com infliximabe, obtendo-se o desaparecimento do quadro articular, regularização do hábito intestinal e a cicatrização das úlceras cutâneas, logo após a primeira infusão. A terapia de resgate com infliximabe foi eficaz para a rápida resposta clínica de paciente com surto agudo de Doença de Crohn e cicatrização de pioderma gangrenoso.

Palavras-chave: Pioderma gangrenoso, Doença de Crohn, Infliximabe.

\begin{abstract}
This article aims to report of a case of gangrenous pyoderma responsive to rescue therapy with infliximab. Female patient, 42 years old, with Crohn's disease using Mesalazine. He evolved with arthralgia in large joints, when Azathioprine was prescribed, and since then he has had phlogistic signs in tissues of both forearms and mucosanguinous diarrhea. He required hospitalization, where antibiotic therapy and then intravenous corticotherapy prevailed, with no improvement in the clinical picture. At the end of the second week of treatment, the cutaneous lesions transformed quite characteristic of gangrenous pyoderma and still had diarrhea. It was biological therapy with infliximab, with the disappearance of the articular condition, regularization of the intestinal habit and healing of skin ulcers, right after the first infusion. The response therapy with infliximab was effective for a rapid clinical response of the patient with acute Crohn's disease and healing of gangrenous pyoderma.
\end{abstract}

Keywords: Gangrenous pyoderma, Crohn's disease, Infliximab.

\footnotetext{
1 Hospital Universitário Getúlio Vargas (HUGV-UFAM), Manaus - AM.

*E-mail: wanderson_loma@hotmail.com
} 
Resumen: Este artículo pretende discutir un caso de pioderma gangrenoso que responde a la terapia de rescate con infliximab. Paciente de 42 años con enfermedad de Crohn que usa mesalazina. Evolucionó con artralgia en grandes articulaciones cuando se prescribió Azatioprina y desde entonces ha presentado signos flogísticos en tejidos blandos de ambos antebrazos y diarrea mucosanguinosa. Requirió ingreso hospitalario, donde inicialmente fue sometido a antibioterapia y posteriormente a corticoterapia intravenosa, sin mejoría de su estado clínico. Al final de la segunda semana de tratamiento, las lesiones cutáneas se volvieron muy características de la pioderma gangrenosa y aún presentaban diarrea. Se inició terapia biológica con infliximab, con desaparición de la afección articular, regularización del hábito intestinal y curación de úlceras cutáneas, inmediatamente después de la primera infusión. La terapia de rescate con infliximab fue eficaz para la respuesta clínica rápida de un paciente con un brote agudo de enfermedad de Crohn y curación de pioderma gangrenoso.

Palabras clave: Pioderma gangrenoso, Enfermedad de Crohn, Infliximab.

\section{INTRODUÇÃO}

O Pioderma Gangrenoso (PG) é uma complicação cutânea grave observada em aproximadamente 1\% dos pacientes com doença inflamatória intestinal (DII) (ARGÜELLES-ARIAS F, et al., 2013). Estima-se que a incidência mundial seja de dois a três casos por 100.000 habitantes por ano, mas isso pode ser subestimado devido à falta de um padrão ouro de diagnóstico (STATES V, et al., 2020).

O PG é uma doença inflamatória e ulcerativa da pele incomum, caracterizada histopatologicamente pelo acúmulo de neutrófilos na pele (dermatose neutrofílica). É importante esclarecer que em contraste com seu nome, o PG não é uma doença infeciosa nem gangrenosa, é uma doença auto-inflamatória. (RODRíGUEZZÚÑIGA MJM, et al., 2019).

A apresentação mais comum do PG é uma pápula ou pústula inflamatória que progride para úlcera dolorosa com borda violácea e a base purulenta. Pode se apresentar também com lesões bolhosas, vegetantes, periestomais e extracutâneas. Mais da metade dos pacientes com PG se desenvolvem em associação com uma doença sistêmica subjacente. Doença inflamatória intestinal, doenças hematológicas e artrite representam as comorbidades mais frequentes (RODRÍGUEZ-ZÚÑIGA MJM, et al., 2019).

A patogênese não é bem conhecida, mas estudos sugerem uma resposta imune anormal em pacientes com predisposição genética, sendo o PG classificado no espectro das síndromes neutrofílicas e autoinflamatórias. Frequentemente ocorre em associação com outras doenças auto-inflamatórias (doença inflamatória intestinal e artrite) (MARZANO AV, et al., 2018).

O diagnóstico do PG é feito através do reconhecimento de achados clínicos e histológicos consistentes e na exclusão de outros distúrbios cutâneos inflamatórios ou ulcerativos. Não existe achados patognomônicos, clínicos ou histopatológicos do PG (RODRÍGUEZ-ZÚÑIGA MJM, et al., 2019).

No tratamento do PG existem várias terapias locais e sistêmicas. Os corticosteroides tópicos e sistêmicos, tacrolimus, ciclosporina e vários produtos biológicos estão entre agentes mais comumente utilizados. Os agentes biológicos direcionados ao Fator de Necrose Tumoral Alfa (TNFa) são os melhores estudados, dada a sua capacidade de tratar outras doenças auto-inflamatórias coexistente (RODRÍGUEZ-ZÚÑIGA MJM, et al., 2019).

Este é um relato de caso sobre PG com descompensação da Doença de Crohn e responsivo a terapia biológica com infliximabe.

\section{DETALHAMENTO DO CASO}

Paciente feminina, 42 anos, natural e procedente do Amazonas, portadora de Doença de Crohn colônica em uso de Mesalazina. Em maio de 2018, evoluiu com artralgia em grandes articulações, quando foi prescrita Azatioprina e desde então iniciou sinais flogísticos em tecidos moles de ambos os antebraços e diarreia mucossanguinolenta. Evoluiu com piora das lesões cutâneas e intensificação do quadro diarreico, necessitando de internação. 
Inicialmente foi prescrita antibioticoterapia via endovenosa com Oxacilina, Ciprofloxacino e Metronidazol e posteriormente, foi acrescentado hidrocortisona $400 \mathrm{mg} /$ dia Via Endovenosa (EV) durante cinco dias, a seguir, prednisona via oral $60 \mathrm{mg} / \mathrm{dia}$. Ao final da segunda semana de tratamento, as lesões cutâneas tornaram-se bem características de pioderma gangrenoso, quando a paciente ainda apresentava diarreia e encontrava-se com anemia ferropriva, hipoalbuminemia e edema periférico acentuado (Figura 1).

Após duas semanas de internação e sem resposta à antibioticoterapia e corticoide EV. Foi iniciado terapia biológica com infliximabe, dose de $5 \mathrm{mg} / \mathrm{kg}$ de peso, nas semanas 0,2 e 6 , obtendo-se cicatrização das úlceras cutâneas, regularização do hábito intestinal, normalização da hipoalbuminemia e desaparecimento do quadro articular e cutâneo logo após a primeira infusão. $O$ exame histopatológico revelou necrose da epiderme ao lado de ulceração e, na derme, infiltrado neutrofílico. A pesquisa de BAAR e a cultura para fungos e bactérias foram negativas.

Figura 1 - Pioderma gangrenoso antes de terapia com infliximabe.

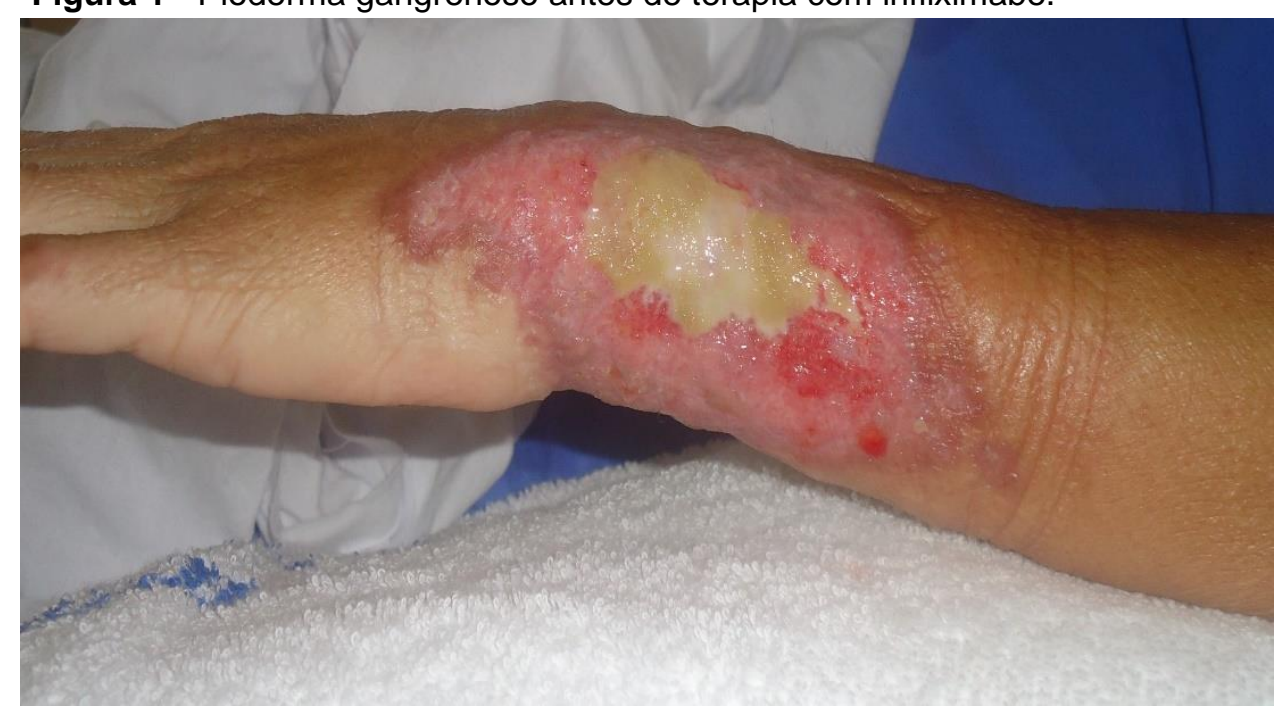

Fonte: Loma WA, et al., 2020.

Atualmente, a paciente encontra-se em manutenção da remissão com infusão de infliximabe a cada oito semanas, e controle da Doença de Crohn e cicatrização completa das lesões do PG (Figura 2).

Figura 2 - Pioderma gangrenoso após a terapia com infliximabe

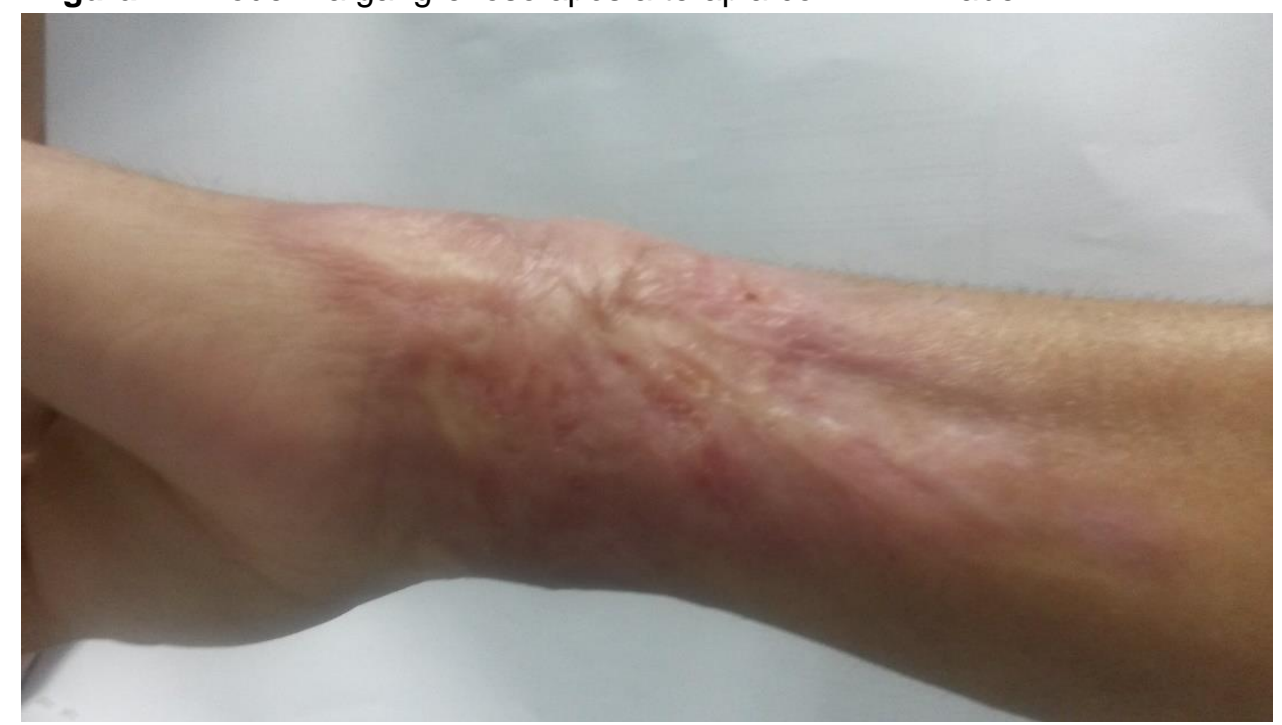

Fonte: Loma WA, et al., 2020. 


\section{DISCUSSÃO}

A apresentação mais comum do PG é a forma ulcerativo, geralmente se inicia como uma ou múltiplas pápulas ou pústulas inflamatórias, muitas vezes precedida de trauma na pele, fenômeno chamado de patergia, que evolui para úlcera dolorosa com borda elevada e delimitada, de progressão lenta e coloração violácea, a base é purulenta que progressivamente aumenta de profundidade e tamanho. A úlcera secreta um exsudato purulento e hemorrágico quando se aplicar pressão nas bordas (RUOCCO E, et al., 2009). O PG ulcerativo, também pode se desenvolver em qualquer área do corpo, incluindo a parede abdominal adjacente ao estoma após colectomia ou cicatrizes cirúrgicas (RODRÍGUEZ-ZÚÑIGA MJM, et al., 2019).

Cerca de metade dos casos de PG tem manifestações extracutâneas relacionadas ou doenças sistêmicas associadas, entre as quais mais comumente incluem doença inflamatória intestinal (DII), neoplasias hematológicas, artrite autoimune e vasculite (NIU R, et al., 2020). Tem incidência estimada de 3 a 10 casos por milhão de pessoas por ano (RUOCCO E, et al., 2009). A lesão cutânea mais comum da DIl é o eritema nodoso em cerca de $4 \%$ dos casos e caracterizado por nódulos subcutâneos dolorosos, com tamanho entre 1 a $5 \mathrm{~cm}$, localização predominantemente em superfícies extensoras das extremidades e parte anterior da tíbia. Geralmente, relacionada com atividade de doença (GREUTER T, et al., 2017).

O PG é a segunda manifestação dermatológica mais comum na DII, ocorrendo em $0,75 \%$ dos pacientes, é mais comum em mulheres, tem importante correlação com a atividade de doença em $50 \%$ dos casos, sendo o seu manejo também benéfico a doença de base (RODRÍGUEZ-ZÚÑIGA MJM, et al., 2019). A fisiopatologia do PG é pouco compreendida e acredita-se envolver desregulação adaptativa e inata do sistema imunológico, anormalidades na função dos neutrófilos (quimiotaxia, adesão), fagocitose anormal e variações genética (ALAVI A, et al., 2017).

As anormalidades na função dos neutrófilos no PG compartilha os mesmos mecanismos pro-inflamatórios encontrados nas síndromes autoinflamatórias. Essas condições são caracterizadas por um sistema imune híper-ativado, levando ao aumento na produção de inflamossomos, responsáveis pela ativação da caspase 1 , uma protease que cliva a pro-interleucina IL-1 $\beta$. O aumento na produção de IL-1 $\beta$ leva a liberação de diversas citocinas pró-inflamatórias, induzindo o recrutamento e a ativação de neutrófilos, que é seguido por uma inflamação mediada por neutrófilos. A IL-17 é indispensável no recrutamento dos neutrófilos na autoinflamação e tem ação sinérgica com o fator de necrose tumoral (TNF) (RODRÍGUEZ-ZÚÑIGA MJM, et al., 2019).

A presença de neutrófilos e células $T$ anormais leva à desregulação imunológica, levando a lesões do PG. Níveis aumentados de mediadores inflamatórios, incluindo IL-1 $\beta$, IL-8, IL-17 e TNF- $\alpha$, causam lesão inflamatória e consequentemente a destruição do tecido, pois aumentam a síntese de metaloproteinases de matriz (MMPs), que são expressadas no infiltrado inflamatório do PG, causando lesão no local, como consequência, a destruição do tecido envolvido, esses mecanismos contribuem para o desenvolvimento da doença, mas ainda existem vários fatores desconhecidos, incluindo o gatilho da desregulação imune e componentes adicionais da imunidade (AHN C, et al., 2018).

A avaliação clínica do paciente com lesão suspeita de PG deve incluir história completa e exame físico. Os pacientes devem ser questionados sobre o histórico de desenvolvimento de lesão, sintomas associados e histórico médico pessoal (MAVERAKIS E, et al., 2018). As manifestações clínicas do PG são variáveis e podem ser divididas em quatro subtipos principais: O PG ulcerativo clássico a variante mais comum explicada anteriormente (RUOCCO E, et al., 2009).

O PG bolhoso (atípico) é uma variante superficial e menos comum do PG que é mais comumente vista em pacientes com $P G$ relacionado à doença hematológica. Ao contrário do $P G$ ulcerativo, os braços e a face são os locais mais comuns de envolvimento. Os pacientes geralmente apresentam rápido desenvolvimento de bolhas inflamatórias azul-acinzentadas nas áreas envolvidas. As bolhas erodem rapidamente, resultando em úlceras superficiais (RUOCCO E, et al., 2009). Devido à forte associação entre PG bolhoso e doença hematológica, os pacientes que se apresentam sem um distúrbio hematológico associado devem ser acompanhados de perto para o desenvolvimento de um distúrbio hematológico (CALLEN JP, et al., 2007).

REAC/EJSC | Vol. 18 | e6368 | DOI: https://doi.org/10.25248/reac.e6368.2021 
O PG pustular comumente se desenvolve em pacientes com doença inflamatória intestinal e com tendência a surgir durante os períodos de exacerbações agudas da doença intestinal. Os pacientes acometidos mostram a rápida evolução de pústulas dolorosas cercadas por eritema. Febre e artralgias concomitantes são comuns (RUOCCO E, et al., 2009).

O PG vegetante é uma forma localizada, isolada e superficial de PG que se apresenta como um nódulo, placa ou úlcera indolente e levemente doloroso com aspecto verrucoso. A cabeça e o pescoço são os locais mais comuns de acometimento (RUOCCO E, et al., 2009).

O PG é considerado um diagnóstico de exclusão, sendo a biópsia essencial na diferenciação de outras etiologias. O melhor local para biópsia é a borda da úlcera ativa. A investigação de histopatologia regular, incluindo coloração especial e cultura de tecidos, é necessária. A histopatologia mostra achados inespecíficos. A existência de inflamação perifolicular, infiltração de neutrofílos e edema são achados inicialmente encontrados em lesões em crescimento ou sem tratamento. O Infiltrado de leucócitos polimorfonucleares tem potencial de levar à formação de abcessos e necrose do tecido (SU WP, et al., 2004).

Achados complementares como hemorragias, trombose secundária de vasos de pequeno e médio calibre e a presença de células gigantes. Vasculite leucocitoclásica secundária é encontrado em cerca de $40 \%$ dos casos. Na imunofluorescência direta são encontrados achados inespecíficos, como deposição de $\operatorname{lgM}$, C3 e fibrina nos vasos da derme papilar e reticular (AHRONOWITZ I, et al., 2012). Devido os achados não possuírem especificidade para PG, as biópsias de pele são uteis para excluir outras causas de ulcerações que podem apresentar achados clínicos semelhantes, tais como neoplasias, vasculites e infecções (SU WP, et al., 2004).

Para que seja feito o diagnóstico de PG, é necessário que estejam presentes um critério principal e dois critérios menores (SU WP, et al., 2004). Os critérios principais são: Progressão rápida de uma úlcera cutânea necrolítica dolorosa com borda irregular, violácea 1 a $2 \mathrm{~cm}$ por dia ou aumento de $50 \%$ no tamanho em um mês; Outras causas de ulceração cutânea foram excluídas (geralmente requer biópsia de pele e investigações laboratoriais) (SU WP, et al., 2004).

Os critérios menores são: História sugestiva de patergia ou achado clínico de cicatriz cribriforme; Doença sistêmica associada a pioderma gangrenoso (doença inflamatória intestinal, artrite); Achados histopatológicos (netrofília dérmica estéril, inflamação mista, vasculite linfocítica); Resposta ao tratamento (resposta rápida ao tratamento com glicocorticoide sistêmico) (SU WP, et al., 2004).

A abordagem cuidadosa de outras doenças que podem causar ulceração cutânea é o primeiro passo importante no tratamento de lesões que parecem com PG (RODRíGUEZ-ZÚÑIGA MJM, et al., 2019). As doença ulcerativas que se parecem ao PG podem ser encontradas em uma de seis categorias: a) Lesão tecidual induzida por drogas; b) Vasculites (poliarterite nodosa, granulomatose de Wegener); c) Doença venosa (SAF, úlcera por estase venosa); d) Neoplasia que envolve a pele (linfoma de células T); e) Infecções cutâneas profundas primárias (esporotricose, tuberculose cutânea); f) Outras doenças inflamatórias (doença de Crohn cutânea) (RODRÍGUEZ-ZÚÑIGA MJM, et al., 2019).

Essa abordagem tem grande importância, uma vez que certos tratamentos utilizados para PG podem ser ineficazes ou prejudiciais em outras doenças. Comumente o tratamento dos pacientes é feito com uma combinação de tratamento tópico e/ou sistêmico que bloqueiam o processo inflamatório e medidas terapêuticas que melhoram a cicatrização da ferida. Embora os sinais iniciais de melhora possam ser evidentes alguns dias após início do tratamento, muitas vezes são necessárias várias semanas a meses para atingir a cicatrização completa da úlcera (RODRÍGUEZ-ZÚÑIGA MJM, et al., 2019).

O tratamento deve ser guiado pela extensão e profundidade da úlcera, doenças sistêmicas associadas, estado clínico do paciente e disponibilidade de medicamentos (RODRÍGUEZ-ZÚÑIIGA MJM, et al., 2019). A monoterapia pode ser usado em pacientes com pioderma gangrenoso leve, pequenas lesões localizadas e em estágios iniciais (pápulas, pústulas, nódulos, poucas úlceras superficiais ou PG vegetante). Quando eficaz, o tratamento local é preferido devido ao risco relativamente baixo de efeitos adversos graves (LE CLEACH L, et al., 2011). 
Na doença mais extensa ou de progressão rápida a maioria dos pacientes com PG não podem ser tratados apenas com terapia local. O tratamento sistêmico é usado como primeira linha no PG que não se limita a algumas úlceras superficiais ou placa solitária de PG vegetante. Agentes sistêmicos também são apropriados para pacientes com PG leve que não melhora com terapia local. Exemplos de agentes típicos de monoterapia incluem esteróides, tacrolimus, cromoglicato de sódio tópico, nicotina, 5-ASA, triancinolona intralesional, ciclosporina intralesional e vários produtos biológicos diferentes são propostos para o tratamento do PG (PATEL F, et al., 2015).

O regime de tratamento de infliximabe, um anticorpo quimérico contra o TNFa, normalmente é administrado $5 \mathrm{mg} / \mathrm{kg}$ nas semanas zero, dois e seis, seguido por infusão a cada oito semanas (CALLEN JP, et al., 2007). Os agentes direcionados ao TNFa são os melhores estudados, dada a sua capacidade de tratar a doença inflamatória intestinal coexistente (PARTRIDGE ACR, et al., 2015).

O estudo de caso supracitado, mostrou a importância do diagnostico, manejo e tratamento do pioderma gangrenoso em uma paciente com Doença de Crohn em atividade. A forma clínica mais comum do pioderma gangrenoso é a ulcerativa, que é mais frequentemente associado a doença inflamatória intestinal, principalmente com a Doença de Crohn. A avaliação e o reconhecimento da lesão no paciente com doença inflamatória intestinal ativa é relevante para o diagnóstico e tratamento, uma vez que a terapia com produtos biológicos tem a capacidade de tratar ambas doenças. A paciente realizou terapia de resgate com infliximabe, essa terapia foi eficaz para rápida resposta clínica da paciente com surto agudo de Doença de Crohn e cicatrização do pioderma gangrenoso.

\section{REFERÊNCIAS}

1. AHN C, et al. Pyoderma gangrenosum: a review of pathogenesis and treatment. Expert Rev Clin. Immunol. 2018; $14(3): 225-233$.

2. AHRONOWITZ I, et al. Etiology and management of pyoderma gangrenosum: a comprehensive review. Am J Clin Dermatol 2012; 13:191.

3. ALAVI A, et al. An Update on Pathophysiology, Diagnosis and Treatment. Am J Clin. Dermatol. 2017;18(3):355-372.

4. ARGÜELLES-ARIAS $F$, et al. Characteristics and treatment of pyoderma gangrenosum in inflammatory bowel disease. Dig Dis Sci. 2013; 58(10): 2949-54.

5. ASHCHYAN HJ, et al. The Association of Age with Clinical Presentation and Comorbidities of Pyoderma Gangrenosum. JAMA Dermatol 2018; 154:409.

6. BRUNSTING LA, et al. Pyoderma (echthyma) gangrenosum: clinical and experimental observations in five cases occurring in adults. Arch Dermatol Syphilol 1930; 22: 655-80.

7. CALLEN JP, et al. Pyoderma gangrenosum: an update. Rheum Dis Clin North Am 2007; 33:787.

8. GREUTER T, et al. Skin manifestations of inflammatory bowel disease. Clinical reviews in allergy \& immunology, 2017; 53(3):413-427.

9. HAGEN JW, et al. Cutaneous manifestations of Crohn Disease. Dermatologic Clinics, 2015; 33(3):417-431.

10. LECLEACH L, et al. Is topical monotherapy effective for localized pyoderma gangrenosum? Arch Dermatol. 2011 Jan;147(1):101-3.

11. MARZANO AV, et al. A comprehensive review of neutrophilic diseases. Clin Rev Allergy Immunol. 2018; 54: 114-130.

12. MAVERAKIS E, et al. Diagnostic Criteria of Ulcerative Pyoderma Gangrenosum: A Delphi Consensus of International Experts. JAMA Dermatol 2018; 154:461.

13. NIU R, et al. Giant pyodermagangrenosum in a patient with ulcerative colitis: A case report. Medicine (Baltimore). 2020;99(6).

14. PATEL F, et al. Effective strategies for the management of pyodermagangrenosum: a comprehensive review. ActaDermVenerol. 2015;95(5):525-31.

15. PARTRIDGE ACR, et al. Effectiveness of systemic treatments for pyodermagangrenosum: a systematic review of observational studies and clinical trials. Br J Dermatol. 2018;179(2):290-295.

16. RODRÍGUEZ-ZÚÑIGA MJM, et al. Pyoderma gangrenosum: a review with special emphasis on Latin America literature. An Bras Dermatol. 2019;94(6):729-743.

17. RUOCCO E, et al. Pyoderma gangrenosum: an updated review. J Eur Acad Dermatol Venereol 2009; $23: 1008$.

18. STATES V, et al. Pyoderma Gangrenosum in Inflammatory Bowel Disease: A Systematic Review and MetaAnalysis. Dig Dis Sci, 2020.

19. SU WP, et al. Pyodermagangrenosum: clinicopathologic correlation and proposed diagnostic criteria. Int $J$ Dermatol. 2004;43(11):790-800.

20. TOLKACHJOV SN, et al. Postoperative pyoderma gangrenosum (PG): The Mayo Clinic experience of 20 years from 1994 through 2014. J Am Acad Dermatol 2015; 73:615. 